\title{
MICOFLORA E QUALIDADE FISIOLÓGICA DE SEMENTES DO ALGODOEIRO TRATADAS COM FUNGICIDAS QUÍMICOS E EXTRATO DE AROEIRA ${ }^{1}$
}

ADALGISA ARANHA DE SOUZA², RISELANE DE LUCENA ALCÂNTARA BRUNO³, EGBERTO ARAÚJO³, GENILDO BANDEIRA BRUNO³

\begin{abstract}
RESUMO - A conscientização ecológica globalizada exige alimentos mais naturais, o que tem levado ao aprimoramento de medidas de controle integrado, através do uso de métodos alternativos para a produção de sementes livres de resíduos tóxicos. Com o objetivo de avaliar a micoflora e a qualidade fisiológica, sementes do algodoeiro (Gossypium hirsutum L.) com e sem línter, cultivares CNPA 7H e Precoce 2 foram tratadas, ou não, com os fungicidas químicos pentacloronitrobenzeno (PCNB), captan, benomyl e tolylfluanid, aplicados puros e também misturados ao extrato de aroeira (Astronium urundeuva L.) e avaliadas quanto à qualidade fisiológica (germinação, índices de velocidade de germinação e de emergência em campo) e à ocorrência de fungos. Utilizou-se o delineamento inteiramente casualizado em esquema fatorial 10x2x2 (tratamentos x cultivares $\mathrm{x}$ tipos de sementes), com quatro repetições. Sementes com línter de ambas as cultivares apresentaram menor qualidade fisiológica. O extrato de aroeira puro não controlou os fungos porém, quando associado aos fungicidas, captan, benomyl e tolylfluanid, reduziu a incidência de Aspergillus spp. e Fusarium sp. na cv. Precoce 2. Houve redução na viabilidade e no vigor das sementes deslintadas tratadas com o extrato de aroeira puro ou associado aos fungicidas. A micoflora foi constituída principalmente por: Aspergillus flavus, Aspergillus sp., Fusarium sp. e Rhizopus sp.
\end{abstract}

Termos para indexação: Gossypium hirsutum L., qualidade fisiológica e sanitária.

\section{MYCOFLORA AND PHYSIOLOGICAL QUALITY OF COTTON SEEDS TREATED WITH CHEMICAL FUNGICIDES AND AROEIRA EXTRACT}

\begin{abstract}
Worldwide ecological awareness requires more natural foods and products which has influenced the improvement and utilization of integrated pest management. In this kind of control, alternative methods are used to protect seeds to decrease the use of chemical products. The purpose of this study was investigate the mycoflora and physiological quality of cotton seeds treated with chemical fungicides and aroeira extract (Astronium urundeuva L.). Cooton seeds (Gossypium hirsutum L.), with or without lint, CNPA 7H and Precoce 2 cultivars, were treated, or not, with the chemical fungicides pentacloronitrobenzeno (PCNB), captan, benomyl and tolylfluanid which were applied pure and mixed with the aroeira extract. The physiological quality was evaluated by germination, speed emergence index, field emergence and fungi presence. The statistical design was completely randomized in a factorial 10x2x2 (treatments x cultivars $\mathrm{x}$ kinds of seeds) with four replications. Cotton seeds, with or without lint, both cultivars showed lower germination and vigour. Pure "aroeira” extract did not control the fungi, but mixed with the chemical fungicides captan, benomyl and tolylfluanid, it showed reduction in the incidence of Aspergillus sp. and Fusarium sp. in the Precoce 2 variety cultivated. There was a reduction in germination and vigour of seeds without lint which were treated with the pure plant extract pure or with combined products. The mycoflora consisted basically of Aspergillus flavus, Aspergillus sp., Fusarium sp. and Rhizopus sp.

Index terms: Gossypium hirsutum L., physiological and healthness quality.
\end{abstract}

\footnotetext{
${ }^{1}$ Aceito para publicação em 06.06.2003; parte da Dissertação de mestrado da primeira autora, para obtenção do título de MSc., na UFPB.

${ }^{2}$ Eng $^{\circ}$ Agr $^{\circ}$, Doutoranda do Programa de Pós - Graduação em Agronomia,
}

Depto. de Fitotecnia, CCA/UFPB, 58397-000, Areia, PB.

${ }^{3}$ Prof $^{\text {es }}$. Adjuntos do Depto. de Fitotecnia, UFPB/CCA; Cx. Postal 22, 58397000, Areia, PB; e-mail: lane@cca.ufpb.br 


\section{INTRODUÇÃO}

O tratamento químico das sementes constitui uma maneira eficiente e econômica de controlar doenças de plantas (Neegaard, 1979). A utilização combinada de fungicidas de amplo espectro tem sido uma das estratégias mais eficazes, permitindo controlar inúmeros patógenos presentes nas sementes e/ou no solo, além de evitar o surgimento de populações resistentes (Goulart, 1998).

Em sementes de algodoeiro, estudos realizados em diferentes regiões do país têm demonstrado a eficiência do tratamento químico. Os produtos tolylfluanid, fludioxanil, etridiazole, prochloraz, imazalil, e o pencycuron, aplicados em formulações e dosagens diversas, reduziram de forma variável e eficiente a incidência de fungos em sementes de algodoeiro, controlando as doenças que causam em condições de campo (Machado, 1996). Além de controlar patógenos nas sementes, a aplicação de fungicidas químicos resultou também na elevação da germinação, conforme foi verificado para os produtos benomyl, thiram (Teixeira et al., 1997), tolylfluanid puro ou misturado a outros produtos (Oliveira et al., 1997), carbendazin + thiram, cardendazin + proclhoraz (Machado et al., 1999), carboxin + thiram (Machado et al., 1999; Patricio et al., 1999; Machado et al., 2000), proclhoraz Mn, metalaxil + iprodione e benomyl + carboxin + thiram (Patricio et al., 1999). Mais recentemente, a eficiência de fungicidas químicos no tratamento de sementes de algodoeiro foi verificada com a aplicação das misturas triadimenol + pencycuron + tolylfluanid, triadimenol + pencycuron e triadimenol + tolylfluanid no controle do tombamento pósemergência (Goulart et al., 2000), e de thiram e tolylfluanid que reduziram em média, $84 \%$ e $76 \%$, respectivamente, do inóculo inicial de Colletotrichum gossypii var. cephalosporioides (Prado et al., 2002).

O tratamento com produtos químicos sintéticos constitui, assim, um método comprovadamente eficiente para o controle de patógenos de sementes. No contexto da agricultura da região Nordeste, verifica-se, porém, para a maioria dos agricultores, a impossibilidade da adoção e/ou o uso correto das práticas de controle químico, podendo advir como conseqüências, problemas ao meio ambiente e à saúde dos operadores. Assim, deve ser levada em consideração que existe, na atualidade, uma conscientização ecológica globalizada que exige uma agricultura "mais natural”. Siqueira (2001) destaca a importância de pesquisas sobre alternativas de controle natural relacionadas à conservação da fauna benéfica e dos inimigos naturais, e tendo em vista sempre o impacto ambiental, uma vez que em média são realizadas entre seis e nove aplicações de defensivos químicos ao longo do ciclo de desenvolvimento do algodoeiro.

O emprego de produtos naturais extraídos da flora nativa poderia constituir-se numa alternativa para melhorar a qualidade das sementes, pois, segundo estudos realizados com o uso de extratos (Khan, 1989; Shetty et al., 1989; Kumar, 1990; Malhotra, 1990; Miah et al., 1990; Magalhães, 1996; Coutinho et al, 1999) e óleos essenciais (Mishra, 1990; Magalhães, 1996), estes foram efetivos no controle de patógenos. Especificamente com relação ao extrato obtido da entrecasca da aroeira (Astronium urundeuva L.), no Estado da Paraíba, esse produto foi usado no tratamento de sementes de feijão Phaseolus vulgaris L. (Coutinho et al., 1999; Felismino et al., 1999), feijão macassar - Vigna unguiculata (L.) Walp. (Magalhães, 1996) e pimentão - Capsicum anuum L. (Macedo, 1997), tendo sido verificado o controle total ou parcial de alguns fungos.

Considerando as possibilidades oferecidas por fungicidas químicos e por produtos naturais e a necessidade de revitalização da cotonicultura dentro do contexto agrícola da região Nordeste, realizou-se o presente trabalho, objetivando avaliar a qualidade fisiológica e sanitária das sementes do algodoeiro herbáceo (Gossypium hirsutum r. L. latifolium Hutch), cv. CNPA-7H e Precoce 2, com e sem línter, tratadas com o extrato de aroeira puro ou associado a fungicidas químicos.

\section{MATERIAL E MÉTODOS}

Foram utilizadas sementes das cultivares CNPA 7H e Precoce 2, produzidas em campos de multiplicação de sementes básicas conduzidos pelo Centro Nacional de Pesquisa do Algodão (EMBRAPA- Algodão). Parte destas sementes foi submetida ao processo de deslintamento químico com ácido sulfúrico na proporção de 3 litros do ácido por 25kg de sementes, sendo posteriormente lavadas em água corrente. Após secagem natural (8 horas) foram classificadas em mesa de gravidade na Usina de Beneficiamento de Sementes (EMEPA-Alagoinha-PB). Em seguida, as sementes (com e sem línter) foram levadas aos Laboratórios de Análise de Sementes (LAS) e de Fitossanidade do CCA-Areia-PB, para serem tratadas (Tabela 1) e, após, avaliadas quanto à qualidade fisiológica e sanitária.

O produto natural (extrato de aroeira) foi cedido pelo Laboratório Fitoterápico da Universidade Estadual da Paraíba, Campina Grande-PB. As sementes foram misturadas aos pro- 
TABELA 1. Produtos e dosagens utilizados no tratamento das sementes de duas cultivares do algodoeiro Gossypium hirsutum L.r. latifolium Hutch, com e sem línter. Areia, PB, 2000.

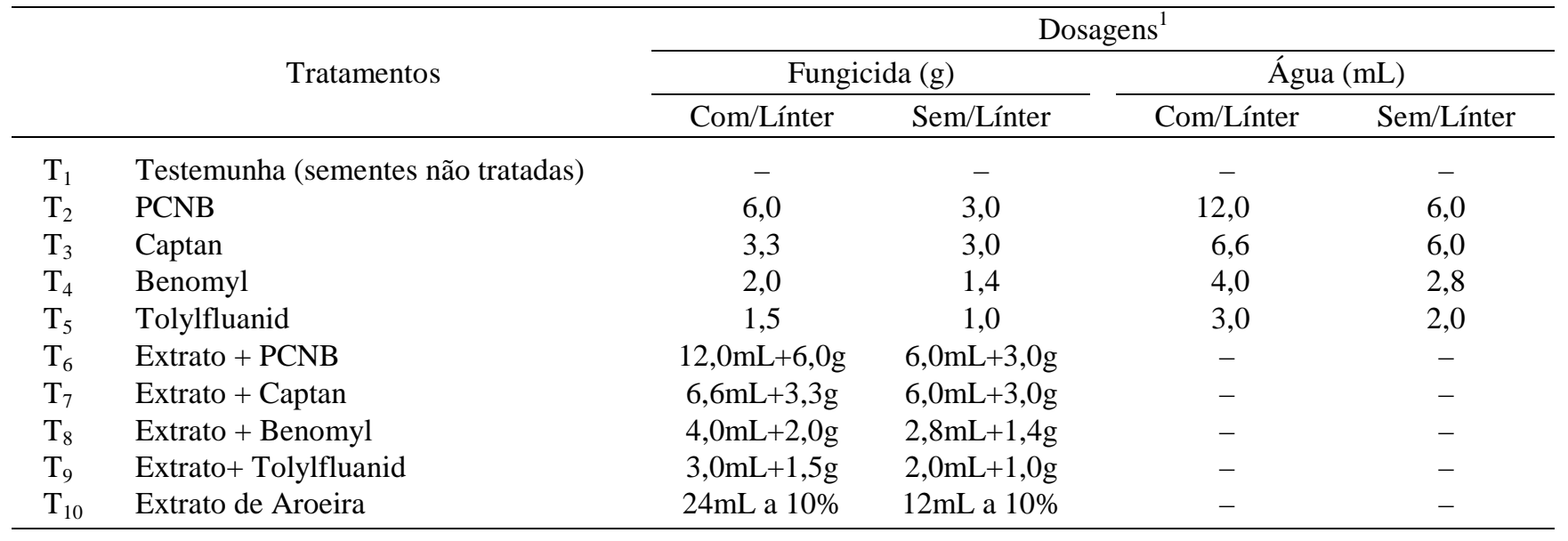

${ }^{1}$ (kg de sementes)

dutos testados, sendo agitadas para melhor aderência dos mesmos. Os fungicidas químicos foram utilizados nas dosagens recomendadas pelo seu fabricante (Tabela 1), proporcional à quantidade de sementes disponíveis; o extrato de aroeira foi diluído em água na proporção de $10 \%$. Após o tratamento, as sementes foram deixadas a secar sob condições não controladas durante 24 horas, para então serem iniciadas as análises laboratoriais.

A qualidade fisiológica e sanitária das sementes foi avaliada pelos seguintes testes: Germinação (TG): quatro repetições de 50 sementes foram colocadas para germinar em rolos de papel toalha "germitest", umedecido com água destilada, em quantidade equivalente a 2,5 vezes o peso do substrato seco, a $25^{\circ} \mathrm{C}$, em presença de luz. A contagem de plântulas normais foi realizada aos quatro e doze dias após o semeio, segundo as Regras para Análise de Sementes (Brasil, 1992) sendo os resultados expressos em porcentagem; Índice de velocidade de germinação (IVG): conduzido juntamente com o teste de germinação. A avaliação de plântulas normais foi realizada diariamente, à mesma hora, a partir da primeira contagem de germinação. O índice foi calculado utilizandose a fórmula proposta por Maguire (1962); Índice de velocidade de emergência em campo (IVEC): quatro repetições de 50 sementes por tratamento foram semeadas em sulcos de $2,5 \mathrm{~m}$ de comprimento, espaçados de $0,20 \mathrm{~m}$, à profundidade de $2 \mathrm{~cm}$, cobertas com aproximadamente $1,5 \mathrm{~cm}$ de solo, mantendo-se a umidade através de irrigações freqüentes. A partir do dia que a primeira plântula emergiu, realizaram-se contagens diárias de plântulas normais até o último dia do teste (21 dias). O índice foi calculado pela fórmula proposta por Maguire (1962); Micoflora das sementes: quatro repetições de 200 sementes para cada tratamento, foram incubadas empregando-se o método "blotter test" (Neergaard, 1979). Estas, em número de dez, foram distribuídas sobre papel de filtro previamente umedecido e esterilizado em placas de Petri e levadas a uma câmara de incubação com temperatura de $24 \pm 2^{\circ} \mathrm{C}$, onde permaneceram sob luz de freqüência próxima ao ultravioleta (NUV) e fotoperíodo de $12 \mathrm{~h}$, durante sete dias. Realizaram-se a identificação e contagem dos fungos, examinando-se as colônias fúngicas desenvolvidas nas sementes sob microscópio estereoscópico. Em alguns casos, a avaliação, foi complementada pela visualização das características morfológicas dos fungos em microscópio óptico comparando-as com as descrições da literatura (Singh et al., 1991). Análise estatística: utilizou-se o delineamento inteiramente casualizado, em arranjo fatorial (10x2x2), com quatro repetições. Os tratamentos consistiram da testemunha e da aplicação de produtos químico e natural (PCNB, captan, benomyl, tolylfluanid, extrato de aroeira e as associações de PCNB+extrato, captan+extrato, benomyl+extrato, tolylfluanid + extrato), duas cultivares (CNPA 7H e Precoce 2) e dois tipos de sementes (com e sem línter). Os dados obtidos em percentagem, referentes ao teste de germinação e micoflora das sementes, foram transformados em arc sen $\sqrt{\mathrm{x} / 100}$. A comparação de médias foi realizada pelo teste de Tukey, a 5\% de probabilidade. Para a análise estatística dos dados foi utilizado o Software Científico NTIA da EMBRAPA (Paniago et al., 1995). 


\section{RESULTADOS E DISCUSSÃO}

O comportamento das cultivares quanto à germinação (Tabela 2A) indicou para a cv. CNPA 7H, que o maior e o menor valor reportaram-se às sementes tratadas com tolylfluanid e extrato de aroeira, respectivamente, apesar deste último não diferir estatisticamente dos tratamentos onde este extrato foi aplicado associado a fungicidas, não diferindo também do benomyl. Por outro lado, para a cv. Precoce 2, o maior valor de germinação foi observado para sementes tratadas com PCNB, que diferiram da testemunha, do extrato puro e em mistura, além do benomyl. Estes resultados estão de acordo com Pozza \& Juliatti (1994), que verificaram semelhante eficiência do tolylfluanid no controle de fungos patogênicos em sementes do algodoeiro, contribuindo para elevar seu po- tencial germinativo. Entre cultivares, em todos os tratamentos avaliados houve maior germinação das sementes da cv. CNPA 7H, com valores equivalentes apenas nas sementes tratadas com PCNB e benomyl.

Ainda com relação à germinação (Tabela 2B), as sementes com línter, tratadas com PCNB, tolylfluanid e extrato de aroeira puro e em mistura, apresentaram as maiores percentagens de germinação; para as sementes deslintadas, os maiores valores de germinação foram os da testemunha e de todos os produtos aplicados isoladamente, que não diferiram entre si. Assim, o uso do extrato de aroeira, nas sementes sem línter foi menos efetivo. Entre os tipos de sementes, aquelas sem línter apresentaram maior germinação quando foram submetidas ao tratamento com fungicidas químicos isolados, o inverso ocorrendo nas sementes com línter. A maior germina-

TABELA 2. Germinação (TG) das sementes de duas cultivares (A) do algodoeiro Gossypium hirsutum L.r. latifolium Hutch, com e sem línter (B), tratadas com fungicidas químicos e extrato de aroeira. Areia, PB, 2000.

\begin{tabular}{|c|c|c|c|c|}
\hline \multirow{3}{*}{ Produtos } & \multicolumn{4}{|c|}{ Cultivares (A) } \\
\hline & \multicolumn{2}{|c|}{ CNPA 7H } & \multicolumn{2}{|c|}{ Precoce 2} \\
\hline & Arcsen $\sqrt{ } \mathrm{x} / 100$ & \multirow{2}{*}{$\begin{array}{c}\% \\
87,25\end{array}$} & Arcsen $\sqrt{ } x / 100$ & \multirow{2}{*}{$\begin{array}{c}\% \\
66,00\end{array}$} \\
\hline Testemunha & 70,70ab A & & 56,10 c d B & \\
\hline PCNB & $68,65 a b$ с A & 85,75 & 68,25 a A & 84,50 \\
\hline Captan & $67,20 a b$ с A & 82,75 & 61,90 ab c B & 75,75 \\
\hline Benomyl & $62,70 \quad$ c d A & 77,25 & 60,30 b c d A & 73,25 \\
\hline Tolylfluanid & $71,00 \mathrm{aA}$ & 88,75 & $66,70 a b$ B & 83,50 \\
\hline PCNB + Extrato & 64,10 b c d A & 80,75 & $53,80 \quad d B$ & 64,75 \\
\hline Captan + Extrato & 64,80 ab c d A & 81,75 & 54,95 & 66,25 \\
\hline Benomyl. + Extrato & $62,90 \quad \mathrm{c} \mathrm{d} \mathrm{A}$ & 79,00 & 57,75 & 70,75 \\
\hline Tolylfluanid + Extrato & $62,20 \quad c$ d A & 78,00 & 54,15 & 65,25 \\
\hline Extrato de Aroeira & 59,90 & 74,75 & 55,55 & 67,75 \\
\hline \multirow{3}{*}{ Produtos } & \multicolumn{4}{|c|}{ Tipos de Sementes (B) } \\
\hline & \multicolumn{2}{|c|}{ Com línter } & \multicolumn{2}{|c|}{ Sem línter } \\
\hline & Arcsen $\sqrt{ } \mathrm{x} / 100$ & $\%$ & Arcsen $\sqrt{ }$ x/100 & $\%$ \\
\hline Testemunha & 51,60 с d B & 60,50 & 75,15 a A & 92,75 \\
\hline PCNB & $61,70 \mathrm{aB}$ & 77,00 & 75,25 a A & 93,25 \\
\hline Captan & 54,30 b c d B & 65,75 & 74,75a A & 92,75 \\
\hline Benomyl & $51,10 \quad d B$ & 60,25 & 71,90a A & 90,25 \\
\hline Tolylfluanid & $64,05 a \mathrm{~B}$ & 80,50 & 73,70a A & 91,75 \\
\hline PCNB + Extrato & $60,65 a b$ A & 75,50 & 57,25 b A & 70,00 \\
\hline Captan + Extrato & $64,70 \mathrm{a} A$ & 81,50 & 55,10 b B & 66,50 \\
\hline Benomyl + Extrato & 64,30 a A & 81,00 & 56,30 b B & 68,75 \\
\hline Tolylfluanid + Extrato & $61,35 a A$ & 76,50 & 55,05 b B & 66,75 \\
\hline Extrato de Aroeira & 58,40 ab с A & 72,25 & 57,10 b A & 70,25 \\
\hline
\end{tabular}

Médias seguidas da mesma letra, minúscula nas colunas $(\mathrm{dms}=6,84)$ e maiúscula nas linhas $(\mathrm{dms}=4,20)$, não diferem significativamente entre si, pelo teste de Tukey a 5\% de probabilidade. 
ção das sementes com línter que receberam aplicação de produtos associados ao extrato de aroeira, se deve, provavelmente, ao línter ter favorecido a maior aderência dos produtos químicos quando em mistura, conforme constatado por alguns autores (Coutinho et al., 1999; Felismino et al., 1999).

Na cv. CNPA 7H, o maior índice de velocidade de germinação se verificou nas sementes tratadas com tolylfluanid, diferindo este tratamento do benomyl, tolylfluanid + extrato e do extrato de aroeira puro (Tabela 3). Nas sementes da cv. Precoce 2, o produto que se sobressaiu foi o PCNB, seguido do tolylfluanid, e do captan. Verificou-se ainda, na maioria dos tratamentos, que a CNPA 7H foi superior à Precoce 2, havendo igualdade entre as mesmas com relação a PCNB, benomyl e tolylfluanid. Já na cv. CNPA 7H, o extrato de aroeira aplicado puro ou misturado, foi estatisticamente igual ao benomyl e captan. Nas sementes deslintadas, ao contrário, todos os tratamentos com extrato de aroeira foram inferiores aos produtos químicos puros e à testemunha.

Para o IVEC (Tabela 4), em todos os tratamentos não houve diferença entre cultivares. Quanto aos tratamentos em

TABELA 3. Índice de velocidade de germinação (IVG) das sementes de duas cultivares do algodoeiro Gossypium hirsutum L.r. latifolium Hutch, com e sem línter, tratadas com fungicidas químicos e extrato de aroeira. Areia, PB, 2000.

\begin{tabular}{|c|c|c|c|c|}
\hline \multirow{2}{*}{ Tratamentos } & \multicolumn{2}{|c|}{ Cultivares } & \multicolumn{2}{|c|}{ Tipos de Sementes } \\
\hline & CNPA 7H & Precoce 2 & Com línter & Sem línter \\
\hline Testemunha & $10,7 \mathrm{ab} A$ & $8,1 \quad \mathrm{c} \mathrm{d}$ e $\mathrm{B}$ & 7,4 b $B$ & 11,5 a A \\
\hline PCNB & $10,5 a b$ с A & $10,3 \mathrm{a} A$ & $9,3 a \quad$ B & 11,5 a A \\
\hline Captan & $10,2 \mathrm{ab}$ c d A & $9,2 \mathrm{ab}$ c B & 7,9 bс B & 11,5 a A \\
\hline Benomyl & 9,5 b c d A & 9,0 b c d A & 7,4 с B & $11,2 \mathrm{a} A$ \\
\hline Tolylfluanid & $10,8 \mathrm{a} A$ & $10,2 \mathrm{ab} \mathrm{A}$ & $9,7 \mathrm{a} \quad \mathrm{B}$ & $11,4 \mathrm{a} A$ \\
\hline PCNB + Extrato & 9,9ab c d A & 7,8 & 9,1ab A & 8,6 b A \\
\hline Captan + Extrato & $10,1 \mathrm{ab}$ c d A & d e B & $10,0 \mathrm{a} \quad \mathrm{A}$ & 8,1 b B \\
\hline Benomyl + Extrato & 9,7ab c d A & c d e B & $9,9 a$ & 8,4 b B \\
\hline Tolylfluanid + Extrato & 9,5 c d A & d e B & $9,2 \mathrm{a} \quad \mathrm{A}$ & 8,2 b B \\
\hline Extrato de Aroeira & d A & c d e B & $8,8 \mathrm{ab}$ A & 8,6 b A \\
\hline
\end{tabular}

Médias seguidas da mesma letra, minúscula nas colunas (dms $=1,22)$ e maiúscula nas linhas $(\mathrm{dms}=0,75)$, não diferem significativamente entre si, pelo teste de Tukey a $5 \%$ de probabilidade.

TABELA 4. Índice de velocidade de emergência em campo (IVEC) das sementes de duas cultivares, do algodoeiro Gossypium hirsutum L.r. latifolium Hutch, com e sem línter, tratadas com fungicidas químicos e extrato de aroeira. Areia, PB, 2000.

\begin{tabular}{|c|c|c|c|c|}
\hline \multirow{2}{*}{ Tratamentos } & \multicolumn{2}{|c|}{ Cultivares } & \multicolumn{2}{|c|}{ Tipos de Sementes } \\
\hline & CNPA 7H & Precoce 2 & Com línter & Sem línter \\
\hline Testemunha & $9,1 \mathrm{ab} A$ & 8,6 b с A & 7,4 b B & 10,3 a A \\
\hline PCNB & 9,5 a A & 9,0 ab c A & $8,0 a b$ B & 10,5 a A \\
\hline Captan & $9,2 \mathrm{a} A$ & $10,1 \mathrm{a} A$ & $8,4 a b$ B & $11,0 \mathrm{aA}$ \\
\hline Benomyl & $9,2 \mathrm{ab} A$ & $9,5 \mathrm{ab} A$ & 7,8ab B & $10,8 \mathrm{aA}$ \\
\hline Tolylfluanid & 8,8ab A & $9,1 \mathrm{abc} \mathrm{A}$ & 7,6ab B & 10,3 a A \\
\hline PCNB + Extrato & $8,2 \mathrm{ab} A$ & 8,2 b c A & 8,5ab A & 7,9 b A \\
\hline Captan + Extrato & 8,9ab A & 8,5 b c A & 9,0 a A & 8,5 b A \\
\hline Benomyl + Extrato & 7,7 b A & 7,8 с A & 7,9ab A & 7,6 b A \\
\hline Tolylfluanid + Extrato & $8,2 \mathrm{ab} A$ & 8,4 b с A & 8,3ab A & 8,3 b A \\
\hline Extrato de Aroeira & $8,4 \mathrm{ab} A$ & 8,1 b с $A$ & 7,8ab A & 8,6 b A \\
\hline
\end{tabular}

Médias seguidas da mesma letra, minúscula nas colunas $(\mathrm{dms}=1,22)$ e maiúscula nas linhas $(\mathrm{dms}=0,75)$, não diferem significativamente entre si, pelo teste de Tukey a 5\% de probabilidade. 
si, da mesma forma como foi verificada para a germinação, as sementes deslintadas tratadas com extrato de aroeira puro ou misturado foram inferiores aos produtos puros e à testemunha.

Considerando o efeito das cultivares com relação a incidência de fungos (Tabela 5) verifica-se, para o Aspergillus flavus, que os resultados da cv. CNPA 7H para os tratamentos testemunha, captan+extrato e extrato de aroeira puro foram superiores aos da Precoce 2. Ainda, para este mesmo fungo, foi constatada para a cultivar CNPA 7H, incidência superior à verificada para os tratamentos com produtos químicos; enquanto na cv. Precoce 2, a maior incidência desse fungo, foi verificada no tratamento com o extrato de aroeira. Para o Aspergillus sp., da mesma forma que o Aspergillus flavus, as incidências nos tratamentos testemunha e extrato de aroeira puro para a cv. CNPA 7H foram superiores à Precoce 2. Constata-se, ainda, com relação a este fungo, maior ocorrência do mesmo nas sementes deslintadas tratadas com o PCNB+extrato e extrato de aroeira puro. Provavelmente, essa ocorrência e a ação patogênica deste fungo, tenham levado à redução da germinação e do vigor das sementes analisadas em ambas as cultivares que receberam estes tratamentos, o que está de acordo com Halloin (1975), quando salienta que, sementes de algodoeiro infectadas com esta espécie fúngica têm sua qualidade fisiológica reduzida mais rapidamente do que outras espécies. Quanto ao Fusarium sp, comparando-se as cultivares (Tabela 5), não foram constatadas diferenças significativas; também, não foram verificadas diferenças entre todos os tratamentos utilizados nas sementes da cv. CNPA 7H, porém, na cv. Precoce 2, a incidência desse fungo nas sementes tratadas com o extrato de aroeira puro foi superior apenas àquelas tratadas com o PCNB, captan+extrato e benomyl+extrato. Assim, fica evidenciada a importância do extrato natural na redução ou inibição dessa espécie fúngica em comparação aos tratamentos utilizados, tanto para cultivares, quanto para tipos de sementes, conforme também constatado por alguns autores (Magalhães, 1996; Coutinho et al., 1999; Felismino et al., 1999). De maneira geral, observou-se maior incidência de Fusarium nas sementes com línter, da cv. Precoce 2, principalmente quando não foram tratadas, provavelmente pela sua susceptibilidade a fusariose, conforme relatos de Di Piero \& Pascholati (2000), além deste fungo ser considerado por vários autores (Pizzinato \& Menten, 1991; Pozza \& Juliatti, 1994) como patogênico para as sementes do algodoeiro.
Em relação às cultivares (Tabela 5), o maior controle do Rhizopus sp., foi obtido em ordem decrescente de eficiência pelos tratamentos, PCNB, captan e tolylfluanid+extrato, nas sementes da cv. CNPA 7H, e nas sementes da cv. Precoce 2, pelo PCNB+extrato. Para as sementes com línter (Tabela 6), a incidência desse fungo contribuiu para a redução da viabilidade e do vigor das mesmas, concordando dessa forma com Halloin (1975) e Lima et al. (1984), ao comentarem que o Rhizopus, quando inoculado em sementes do algodoeiro, afeta de imediato o vigor, estando provavelmente associado ao apodrecimento da semente durante o início do processo de germinação. Nas sementes tratadas com o extrato de aroeira puro, as incidências de todos os fungos foram superiores aos demais tratamentos, exceto para o Aspergillus sp. nas sementes com línter e para o Fusarium sp. nas sementes deslintadas. As incidências nas sementes sem línter, nos tratamentos que foram constituídos por fungicidas químicos, foram semelhantes entre si e à testemunha, exceto quanto ao Aspergillus flavus; nas sementes com línter, a efetividade dos tratamentos variou conforme a espécie fúngica assinalada.

Com relação à testemunha, a maior ocorrência de fungos foi detectada nas sementes com línter, o que está de acordo com Queiroga \& Beltrão (1997) quando informam a importância do uso de sementes deslintadas; por outro lado, Soave (1985) também recomenda o uso do deslintamento em sementes do algodoeiro, salientando que o línter abriga cerca de $70 \%$ dos fungos encontrados nas sementes, indicando que o deslintamento, por si só, já melhora a qualidade sanitária dos lotes, como também a sua germinação.

\section{CONCLUSÕES}

O extrato de aroeira, aplicado puro ou associado aos fungicidas químicos reduziu a qualidade fisiológica das sementes deslintadas;

As sementes com línter, tratadas com o extrato de aroeira associado aos fungicidas captan, benomyl e tolylfluanid alcançaram maior viabilidade e vigor (IVG);

As espécies fúngicas de maior incidência, em ambas as cultivares, especificamente nas sementes com línter foram Aspergillus flavus, Rhizopus sp. e Fusarium sp.

O extrato de aroeira puro não controlou os fungos, porém, quando associado aos fungicidas captan, benomyl e tolylfluanid, reduziu a incidência de Aspergillus spp. e Fusarium sp. nas sementes da cv. Precoce 2. 

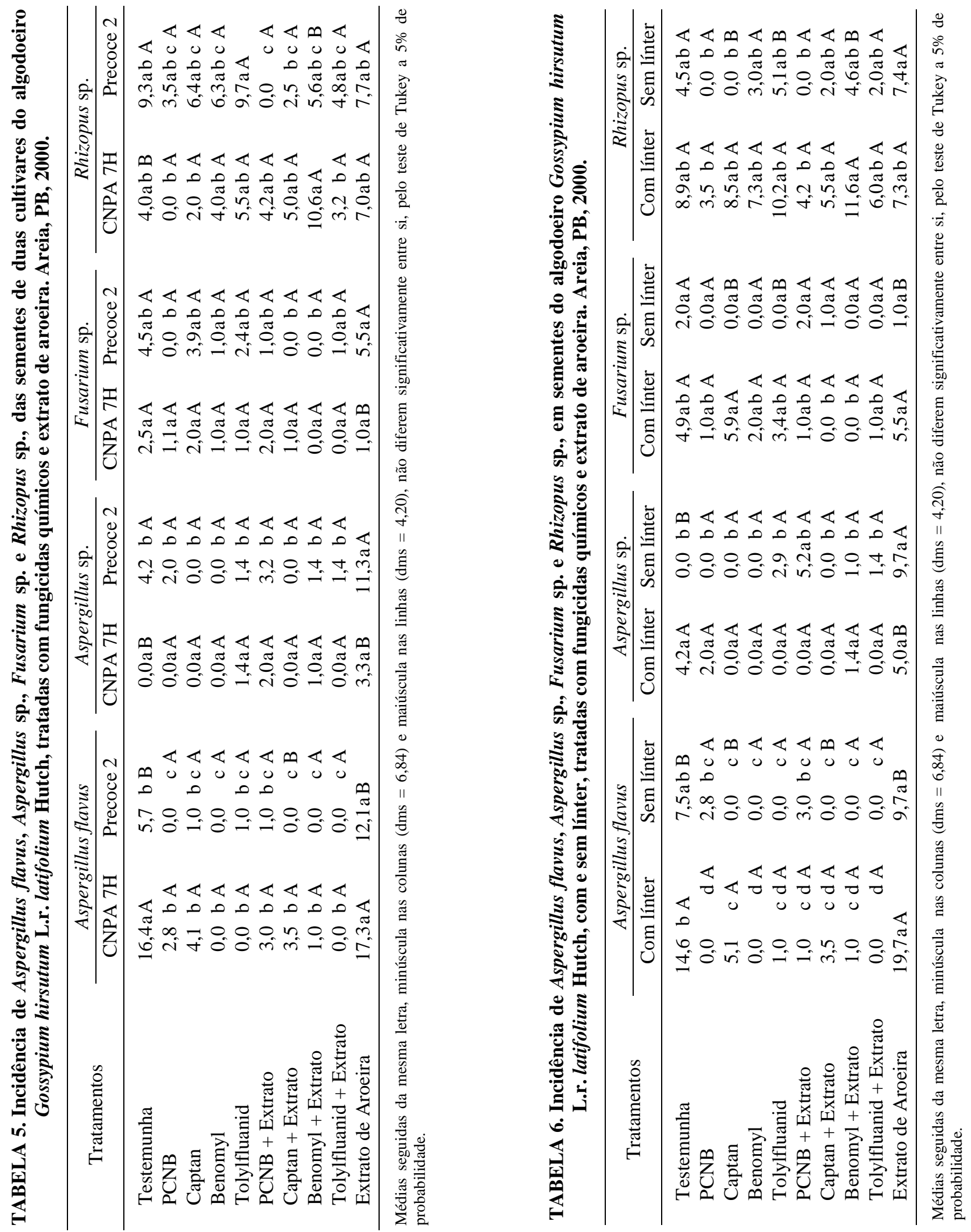


\section{REFERÊNCIAS}

BRASIL, Ministério da Agricultura. Regras para análise de sementes. Brasília: SNDA/DNDV/CLAV, 1992. 365p.

COUTINHO, W.M.; ARAÚJO, E.; MAGALHÃES, F.H.L. Efeito de extratos de plantas anacardiáceas e dos fungicidas químicos Benomyl e Captan sobre a micoflora e qualidade fisiológica de sementes de feijão (Phaseolus vulgaris L.). Ciência e Agrotecnologia, Lavras, v.23, n.3, p.560-568, 1999.

DI PIERO, R.M.; PASCHOLATI, S.F. Produção de celulases por Fusarium oxysporium f. sp. vasinfectum e seu papel na patogenicidade em algodoeiro. Summa Phytopathologica, Jaguariúna, v.26, n.3, p.336-341, 2000.

FELISMINO, D.C. et al. Qualidade fisiológica e sanitária de sementes de feijão (Vigna unguiculata (L.) Walp. e Phaseolus vulgaris L.) tratadas com produtos químicos e naturais e armazenadas em ambiente não controlado. Revista Brasileira de Sementes, Brasília, v.21, n.1, p.198-207, 1999.

GOULART, A.C.P. Tratamento de sementes com fungicidas. In: EMBRAPA. Centro de Pesquisa Agropecuária do Oeste. Algodão: informações técnicas. Dourados, 1998. p.71-84. (EMBRAPA. CPAO. Circular Técnica, 7).

GOULART, A.C.P.; ANDRADE, P.J.M.; BORGES, E.P. Controle do tombamento de plântulas do algodoeiro causado por Rhizoctonia solani pelo tratamento de sementes com fungicida. Summa Phytopathologica, Jaguariúna, v.26, n.3, p.362-368, 2000.

HALLOIN, J.M. Solute loss from deteriorates cottonseed: relationship between deterioration, seed moisture and solute loss. Crop Science, Madison, v.15, p.11-15, 1975.

KHAN, M.I. Effect of Nicotiana spp on seed mycoflora of wheat, Indian Journal of Applied and Pure Biology, Bophal, v.4, n.1, p.69-71, 1989.

KUMAR, S. Performance of leaf extracts preservation of padaly seed. Seed Research, New Delhi, v.18, n.1, p.95-97, 1990.

LIMA, E.F.; VIEIRA, R.M.; CARVALHO, J.M.F.C. Influência de Rhizopus sp, A. niger e A. flavus na deterioração de sementes de algodoeiro armazenadas. Fitopatologia Brasileira, Brasília, v.9, n.3, p.555-560, 1984.

MACEDO, L.P.M. Micoflora e germinação de sementes de pimentão (Capsicum annuum L.) tratadas com produtos químicos e óleo de piqui (Cariocar brasiliensis Camb.). 1997. 26f. Trabalho (Graduação) - Universidade Federal da Paraíba, Areia.

MACHADO, J.C. Tratamento de sementes de algodão visando controle de patógenos. In: SIMPÓSIO BRASILEIRO DE PATOLOGIA DE SEMENTES, 4., Campinas, 1996. Anais ... Campinas: Fundação Cargill, 1996. p.69-76

MACHADO, A.Q. et al. Controle de patógenos associados às sementes de algodoeiro. In: CONGRESSO BRASILEIRO DO ALGODÃO, 2., 1999, Ribeirão Preto. Anais... Campina Grande: EMBRAPA, 1999, p. 481-482.

MACHADO, A.Q. et al. Controle de fungos e desempenho inicial de sementes de algodão tratadas com carboxin + thiram. In: CONGRESSO BRASILEIRO DE FITOPATOLOGIA, 33., Brasília, 2000. p. 389.
MAGALHÃES, F.H.L. Efeito dos óleos de piqui (Cariocar brasiliensis Camb), dendê (Elaeis guianensis L.), dos fungicidas químicos Benomyl e Captan sobre a microflora e qualidade fisiológica de sementes de feijão (Phaseolus vulgaris L). 1996. 32f. Trabalho ( Graduação) - Universidade Federal da Paraíba, Areia.

MALHOTRA, D.; RAI, P.K. Observations on the effect leaf on germination and fungi associated whith seeds of Glycine max L. Indian Journal of Applied and Pure Biology, New Delli, v.5 n.1, p. 53 - 56, 1990.

MAGUIRE, J.D. Speed of germination-aid in selection and evaluation for seedling emergence and vigour. Crop Science, Madison, v.2, n.1, p.176-177, 1962.

MIAH, M.A.T. et al. Anti-fungal activity of some plant extracts. Bangladesh Jurnal of Botany, Dacca, v.19, n.1, p.5-10, 1990.

MISHRA, D. Seed protectant property of essential oil Zingiber officinale Roscal. Indian Perfumer, New Delli, v.4, p.266-268, 1990.

NEERGAARD, P. Seed pathology. 2.ed. London: Mac Millan Press, 1979. v.2, 1191p.

OLIVEIRA, J.A.; MACHADO, J.C.; VIEIRA, M.G.C.C. Efeito do tratamento fungicida em sementes de algodoeiro visando controle de tombamento causado por Colletotrichum gossypii. In: CONGRESSO BRASILEIRO DE FITOPATOLOGIA, 30., 1997, Poços de Caldas. v.22, p.291.

PANIAGO, C.F.A. et al. Software científico NTIA. Campinas: EMBRAPA - NTIA, 1995 . v.1

PATRÍCIO, F.R.A. et al. Tratamento de sementes de algodão com fungicidas. Summa Phytopathologica, Jaguariúna, v.25, n.3, p.250256, 1999.

PIZZINATO, M.A.; MENTEN, J.O.M. Patogenicidade de oito espécies de Fusarium, isoladas de sementes, às plântulas de algodoeiro. Summa Phytopathologica, Jaguariúna, v.17, p.124-134, 1991.

POZZA, E.A.; JULIATTI, F.C. Tratamento de sementes no controle de doenças iniciais do algodoeiro. Fitopatologia Brasileira, Brasília, v.19, n.3, p.384-389, 1994.

PRADO, P.E.R. et al. Eficácia do tratamento químico de sementes de algodoeiro em relação ao potencial de inóculo de Colletotrichum gossypii var, cephalosporioides. In: SIMPÓSIO BRASILEIRO DE PATOLOGIA DE SEMENTES, 7., 2002, Sete Lagoas. Anais... p.52.

QUEIROGA, V.P.; BELTRÃO, N.E.M. Avaliação da qualidade fisiológica das sementes de algodão submetidas aos fatores colheita vs beneficiamento de descaroçadores. In: CONGRESSO BRASILEIRO DO ALGODÃO, 1., 1997, Fortaleza. Anais... Campina Grande: EMBRAPA, 1997. p.576-579.

SHETTY, S.A.; PRAKASH, H.S.; SHETTY, H.S. Efficacy of certain plant exctracts against seed-borne infection of Trichoconiella padwickii in padaly (Oriza sativa). Canadian Journal of Botany, Otawa, v.77, n. 7, p.1956-1958, 1989.

SINGH, K. et al. An illustrated manual on identification of some seed-borne Aspergilli, Fusaria, Penicillia and their mycotoxins. Hellerup, Copenhagen: Danish Government Institute of Seed Pathology for Developing Countries, 1991. 133p. 
SIQUEIRA, F.V. Desafio é reduzir volume de defensivo. Anuário Brasileiro do Algodão, p.118, 2001.

SOAVE, J. Diagnóstico da patologia de sementes de algodoeiro no Brasil. Revista Brasileira de Sementes, Brasília, v.7, n.1, p.195201, 1985.
TEIXEIRA, H., MACHADO, J.C.; CARVALHO VIEIRA, M.G.G. Avaliação dos efeitos do tratamento algodoeiro Gossypium hirsutum L.r. latifolium Hutch, com e sem línter. Areia, PB, 2000.químico e biológico na transmissão de Colletotrichum gossypii South. em sementes de algodoeiro (Gossypium hirsutum L.). Ciência e Agrotecnologia, Lavras, v.21, n.4, p.413-418, 1997. 